\title{
Network Engineering Project Design of Teaching Based on Process Simulation
}

\author{
Fang Fang \\ Network Engineering and Research Center, \\ South China Univ. of Technology, \\ Guangzhou, China, 510641 \\ ffang@scut.edu.cn
}

\author{
Yiqin $\mathrm{Lu}$ \\ Network Engineering and Research Center, \\ South China Univ. of Technology, \\ Guangzhou, China, 510641 \\ eeyqlu@scut.edu.cn
}

\begin{abstract}
A new teaching mode for network design is proposed, which is based on simulating the process of network engineering. The teaching mode can help students to master the necessary designing skills of both small Enterprise LANs and WANs. By simulating the upgrade of networks of a cultural center, ,the details of design processes are exhibited, such as collecting customer requirements, translating those requirements into equipment and protocol needs, and creating a network topology which addresses the needs of the customer. Students are familiarized with how to create and implement a design proposal for a virtual customer. This kind of teaching mode prepares the students with the skills required for entrylevel Pre-Sales Support and entry-level Network Design jobs.
\end{abstract}

Keywords- Network Engineering; Simulation; Network Design; Teaching Mode

\section{INTRODUCTION}

Traditional curriculum system emphasizes the guidance of the theory. In this training mode, talents have deep theoretical foundation, suitable for the cultivation of Academic and research talent. But the industry is in favor of the practical operation, and requires strong engineering capabilities. [1]

Computer network courses have a lot of professional orientations, the course discussed in this paper focused on net-work engineering, including the following three capabilities:

- Ability of building network. According to actual needs, planning the network layout, and by the reasonable application of the technology, set up to meet the practical requirements of the network.

- Ability of the network maintenance. Network maintenance is no longer just to keep the connection, but according to the characteristics of different regions to management.

- Ability to solve problems. Many factors influence the network. How can the first time by means of analysis of protocol data packets, finding the problem source is crucial. [5]

\section{NETWORK DESIGN PRINCIPLE AND METHODS}

Network designers ensure that our communication networks are able to adjust and scale to the demands for new services. The four fundamental technical requirements of network design are: Scalability, Availability, Security and Manage-ability. Using hierarchical network design principles and an organized design methodology, designers create networks that are both manageable and supportable. [3]

\section{TEACHING CASE}

According to the learn cognitive process, from basic to professional, from simple to comprehensive, network engineering experiments can generally be divided into:

- Basic experiment: The main purpose is to cognitive and verification, and master the basic configuration of equipment, verify configuration effect.

- Comprehensive experiment: Comprehensive knowledge of different chapters.

On this basis, teachers add the teaching content of the simulation network: network design in accordance with the process of project implementation and specification, organize students into groups for collaboration, co-design, the form of teamwork to complete a project. The simulation project covers the network core technology, such as LAN, WAN, wireless network, network security, network management and network applications. [2]

\section{A. Design the background of the case}

Simulate a CultureCompany, it manages a cultural center, main business scope includes: the investment in construction and operational management of the cultural center, organizing various cultural performances, conferences, exhibitions, performing arts supplies sales business. To meet business growth needs, The CultureCompany plans to upgrade the data network of cultural centers.

In the case of this experiment, the role of students is the network designer responsible for preparing the requested documents of this network upgrade project.

Network design projects are normally divided into the following contents:

- Identifying Network Requirements

- Designing the Network Topology and solutions

- Verification tests

- Preparing the proposal of project

\section{B. Identifying Network Requirements}

1) Identifying Technical Requirements and Constraints

The total construction area of the cultural center is 120,000 square meters. The Cultural center has 20000 seats, 50 luxury VIP rooms and 1000 parking Spaces. The function of the cultural center from the stage shows, to concerts, exhibitions, sporting events and other various activities can be held. The CultureCompany hopes that through this 
network upgrades, which can improve the customer experience and provide more kinds of services. In addition to the performance venues, as well as offices, ticket offices, exhibition halls, shops and restaurants, the cultural center will also provide video services for events and civic performances.

Teachers create a network organization structure of the CultureCompany. It includes all stakeholders in the structure - internal network users, IT organizations, external customers, suppliers, and partners. Teachers also describe the roles of each member of the network partner team: Account Manager, Pre-sales Systems Engineer, Network Designer, and Postsales Field Engineer. [3]

First, Students need use prioritized business goals to determine technical requirements for the network upgrade project. Students will create and prioritize the technical requirements for the network so that it meets the CultureCompany business goals and priorities. Teachers require students to use the CultureCompany business goals to create and prioritize the technical requirements for the network. Students list the ranked technical requirements in a table and assign a priority value as a percentage.

TABLE I. THIS IS AN EXAMPLE OF TECHNICAL REQUIREMENTS FOR THE CULTURAL CENTER NETWORK DESIGN PROJECT

\begin{tabular}{|c|c|c|}
\hline Category & Technical Requirements & Priority \\
\hline Availability & $\begin{array}{c}\text { E-commerce, security, and IP } \\
\text { telephony systems rely on the } \\
\text { underlying network being available } 24 \\
\text { hours a day, 7 days a week. }\end{array}$ & $40 \%$ \\
\hline Security & $\begin{array}{c}\text { Increase the firewall, and make sure } \\
\text { that the server and wireless security. }\end{array}$ & $30 \%$ \\
\hline Scalability & $\begin{array}{c}\text { Wireless coverage of the entire venue } \\
\text { in the future; With the increase in the } \\
\text { number of users, the network system } \\
\text { to facilitate the expansion. }\end{array}$ & $20 \%$ \\
\hline Manageability & $\begin{array}{c}\text { To use the management and } \\
\text { monitoring software }\end{array}$ & $10 \%$ \\
\hline & Total & $100 \%$ \\
\hline
\end{tabular}

Second, Students need identify how constraints imposed by the customer affect the design of the network. Teachers require students to develop a list of possible constraints that set limits on the network upgrade project by brainstorming ideas with other students.

TABLE II. THIS IS AN EXAMPLE OF CONSTRAINTS FOR THE CULTURAL CENTER NETWORK DESIGN PROJECT

\begin{tabular}{|c|c|c|}
\hline CONSTRAINT & GATHERED DATA & COMMENTS \\
\hline Budget & $\begin{array}{c}\text { Project funds have been } \\
\text { implemented }\end{array}$ & $\begin{array}{c}\text { Software and } \\
\text { hardware upgrade } \\
\text { step by step }\end{array}$ \\
\hline Policy & $\begin{array}{c}\text { The company request to } \\
\text { purchase international well- } \\
\text { known brand product. Need to } \\
\text { buy hardware equipment } \\
\text { maintenance services. }\end{array}$ & $\begin{array}{c}\text { Does not exceed the } \\
\text { scope of budget }\end{array}$ \\
\hline Schedule & $\begin{array}{c}\text { To complete the project within } \\
\text { six months }\end{array}$ & $\begin{array}{c}\text { Implementation of } \\
\text { the upgrading } \\
\text { necessary to avoid } \\
\text { performing frequent } \\
\text { periods (such as the } \\
\text { New Year festival) }\end{array}$ \\
\hline Personnel & $\begin{array}{c}\text { Design team need } \\
\text { extra manpower }\end{array}$ \\
\hline
\end{tabular}

\section{2) Characterizing the Existing Network}

One of the first tasks the designer does when characterizing the network is to ensure that the network documentation and topology diagrams are up-to-date.

The network designer examines the existing network to determine if the design goals are realistic and feasible, and if the current network can meet the expectations for scalability, availability, security and manageability. At this stage, the students need to complete the following tasks:

- Characterize an existing network to identify strengths and weaknesses present in the design.

- Choose the appropriate hardware and software upgrades to prepare the network for the integration of new technologies.

- Create a detailed network Design Requirements document.

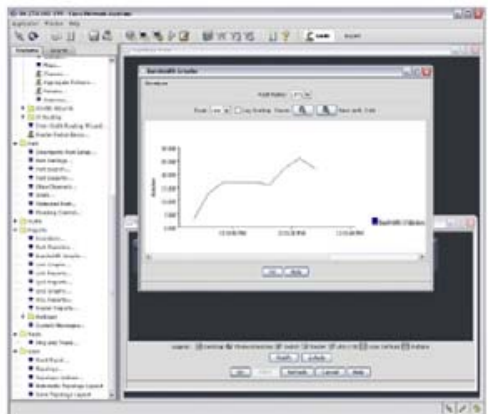

Figure 1. Cisco Network Assistant's monitoring graphics[3]

Teachers suggest students to use the monitoring software CAN (Cisco Network Assistant). It is used to note if baseline performance is exceeded. This information will help determine how the Culture centre network needs to be upgraded to meet the requirements of the CultureCompany.

3) Identifying Application Impacts

The important applications of the cultural centre include: e-commerce, real-time video, security monitoring, etc. In the network without QOS (quality of service), all packets are treated equally, and Real-time application performance will be affected.

The success of a network upgrade depends on meeting the goals of the network users. End users evaluate the performance of the network based on the availability and responsiveness of their applications. Application traffic types and the mix of applications on the network determine the bandwidth and throughput requirements of the network design. The primary goal of QOS is to provide priority, including dedicated bandwidth, controlled jitter and latency, and reduced packet loss. QOS does not actually create more bandwidth. QOS uses traffic queues to manage bandwidth use to support the applications, which are the most sensitive to delays. There are three basic steps to ensure that traffic is properly prioritized: [3]

- Step 1: Identify Traffic Requirements

- Step 2: Define Traffic Classes

- Step 3: Define QOS Policies 
Teachers require students to rank traffic based on given scenarios specific to the cultural centre case study. Students set up a Priority Queue to mark packets, and use NetFlow to view packet markings. Figure 2 is an example of a QOS configuration, set up three different priority applications.

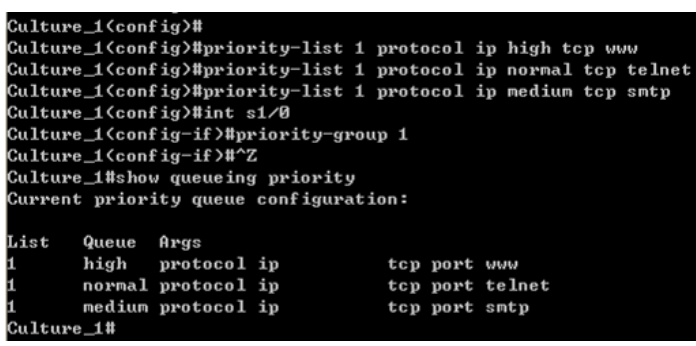

Figure 2. An example of QoS configuration

\section{Designing the Network Topology and solutions}

It is critical to the success of a network design project that all of the business goals are addressed with the new design. Determining which elements to include in the design is a multistep process. At each step of the process, the network designer takes into account the goals and requirements, the existing network capabilities and the new technologies that need to be integrated. The produce the final design, the network designer choose the best equipment and technology solutions to meet the goals and requirements of the customer.

In this part of the experiment, the students design team analysis cultural center's business objectives and technology requirements, and complete the following tasks.

1) Design the Core, Distribution and Access Layer topologies for a campus network [3]

- At the Core Layer, the elements of the design include: redundant components and links, high availability features, and fast converging protocols.

- Distribution Layer design elements include: redundant components and links, high density routing, traffic filtering, QOS mechanisms, fast convergence and traffic aggregation.

- Design elements required at the Access Layer include: port density, VLAN strategies, physical security, power requirements, QOS classification and marking capabilities, and the support for redundant links to the Distribution Layer.

The following figure is an available reference topology for students. Teacher should require students to discuss their topology design according to the requirements made earlier.

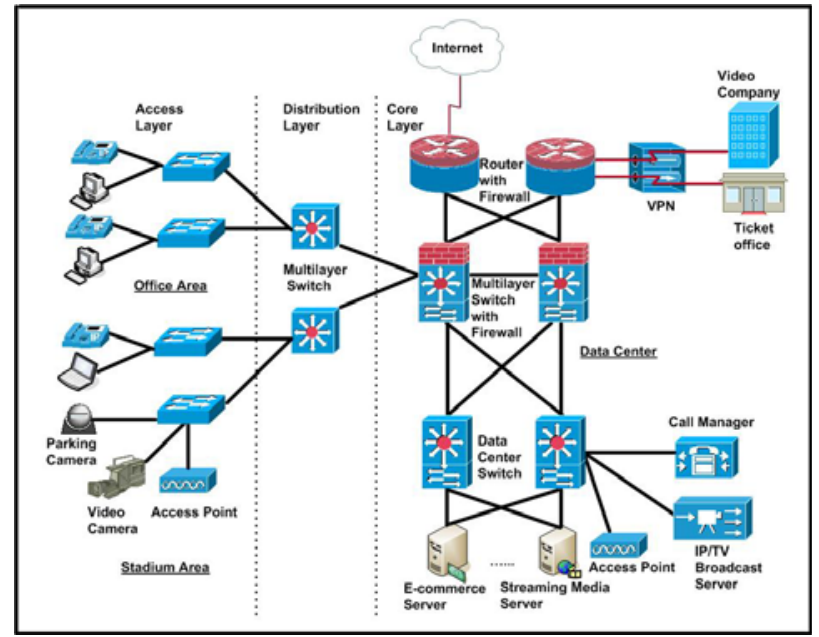

Figure 3. Reference topology of the cultural center

2) Design the WAN connectivity module and remote worker support

A Video Production Company hired to provide video during and after events, needs to connect to the cultural center network to exchange files. Designing WAN connectivity requires evaluation of the telecommunications services available in the customer's geographic area. Because WAN connectivity may not be as reliable as LAN connectivity methods, it is important to consider implementing back-up or alternate access methods in a WAN design.

VPN (Virtual Private Network) can provide secure remote access through the Internet for remote workers and remote ticket offices. This type of VPN connection is also an effective backup connectivity option.

3) Design wireless network

In order to meet the goal of the cultural centre network design, wireless network will coverage in the following areas: exhibition halls, restaurants, VIP rooms. The availability of a wireless connection is dependent on the following factors: Location of the AP; Signal strength of the AP; Number of users sharing the AP connectivity. For management purposes, in the case of the cultural centre, the design team suggested the use of wireless controllers.

4) Incorporate security into the network design

The network designer must identify which data and communications are at risk and what the potential sources of attacks are. Security services then need to be placed at appropriate points throughout the network design to prevent likely attacks. The e-commerce servers on the cultural centre network contain customer information that may include credit card and banking details. The cultural centre's servers contain personnel and payroll information. These servers and the infrastructure that transports the data they contain must be secured adequately to protect this information from unauthorized use. Security measures relating to the cultural centre's wireless network need to be considered as well. For the safe deployment of the cultural centre, students should focus on the following aspects: [3] 
- Provide physical security for the infrastructure devices,

- Prevent unauthorized users from accessing the network.

- Use the firewall and IDS (Intrusion Detection System) to protect against threats and attacks.

\section{Verification tests}

Performing testing of a new design before deployment reduces risk and shortens implementation time. The verification tests demonstrate new or improved functionality of the network, as well as verifying the design works as expected., When it is not possible to duplicate the physical network environment in order to perform a verification test, it may be necessary to use a simulation software program to test the design. Verification tests can be used to identify risks and weaknesses inherent in the network design. Some commonly identified risks and weaknesses include: Single points of failure; possible bottlenecks; limited scalability; overly complex designs. [3]

\section{E. Preparing the proposal of project}

Organizing the network design data into a formal presentation requires the skills of the entire design team. Presenting the proposal to the customer and obtaining an agreement to implement the proposed network represents the successful conclusion of the design project.

A proposal of network project typically contains the following sections: [3]

- Executive Summary: The goal of the summary is to convince the CultureCompany decision-makers of the business benefits of the design.

- Network Requirement: Review the business goals and network requirements, including users and applications that need to be supported.

- Current Network Environment: Document the state of the existing cultural center network. It summarizes the results of the network characterization, including strengths and weaknesses of the existing network.

- Proposed Physical Design: Describe the physical layout of the proposed design. The section describes the features and recommended uses for the technologies and devices proposed for the new network design. This section documents the new Culture center data center.

- Proposed Logical Design: Describe the logical topology of the proposed network. The section describes the routing and switching protocols recommended for the planned network, including recommended security mechanisms and products that support the security policy of the business.

- Implementation Plan: Provides a detailed list of the tasks that must be performed to install and implement the new network. This section includes tasks, steps, time required, and proposed schedules.

- Cost Proposal: Provides cost proposal for equipment, software, installation, and ongoing support.

\section{CONCLUSION}

By simulating a complete project of a network upgrade, the students will clearly understand the implementation of network engineering. The design process can consolidate not only the students' mastery of the methods of equipment installation and systems configuration, but also their understanding of the concept of system cooperation. [4] This article simulated the project of a cultural centre. In actual teaching, the teacher can consider more kinds of application cases.

\section{REFERENCES}

[1] Cai Wandong; Zhang ShengBing. Reforming computer network curriculum to improve the student $s$ engineering ability, Experimental Technology and Management, 2008(12): pp.24-25.

[2] Cao JieNan, Xu Ming, Zhao WenTao, Cai ZhiPing, , Network engineering experiments to decomposition and integrated, China Education Network, 2008(11): pp.64-66.

[3] http://www.cisco.com/web/learning/netacad/index.html

[4] Cao JieNan, Xu Ming, Zhao WenTao, Cai ZhiPing, , Research and practice of network engineering experimental teaching, Computer Education, 2008(20):pp.11,35-37.

[5] http://www.ruijie.com.cn/education.aspx/ 